Pirineos. Revista de Ecología de Montaña

Vol. 175

Jaca, Enero-Diciembre, 2020, e055

ISSN-1: 0373-2568

https://doi.org/10.3989/pirineos.2020.175005

\title{
FENOLOGÍA DE LOS ECOSISTEMAS DE ALTA MONTAÑA EN ANDALUCÍA: ANÁLISIS DE LA TENDENCIA ESTACIONAL DEL SAVI (2000-2019)
}

\section{Phenology of the high-mountain ecosystems in Andalusia: Seasonal trend analysis of SAVI (2000-2019)}

\author{
Oliver Gutiérrez-Hernández \\ Departamento de Geografía. Universidad de Málaga.
}

Identificador ORCID de autor y e-mail:

Oliver Gutiérrez-Hernández: http://orcid.org/0000-0003-2580-5465. E-mail: olivergh@uma.es

\begin{abstract}
Recibido: 27-04-2020. Aceptado: 05-06-2020. Fecha de publicación on.line: 02/09/2020
Citation / Cómo citar este artículo: Gutiérrez-Hernández, O. (2020). Fenología de los ecosistemas de alta montaña en Andalucía: Análisis de la tendencia estacional del SAVI (2000-2019). Pirineos, 175, e055. https://doi.org/10.3989/pirineos.2020.175005

RESUMEN: Los paisajes vegetales de alta montaña constituyen un singular ecosistema en la región más meridional de la península ibérica, donde la estación favorable coincide con un periodo de sequía. En este trabajo, se usaron las imágenes de satélite del sensor Terra-MODIS para desarrollar un análisis de tendencia estacional basado en el cálculo del Índice de Vegetación Ajustado al Suelo (SAVI) de 460 imágenes de satélite con frecuencia de 16 días y aplicado sobre las superficies cubiertas por las comunidades vegetales de los pisos oromediterráneo y crioromediterráneo en Andalucía, con el fin de obtener las curvas de tenencia estacional modeladas (o ajustadas) para el primer (2000) y último (2019) año de la serie. Los resultados obtenidos permitieron caracterizar la curva fenológica típica de estos ecosistemas, con mínimos en invierno (febrero) y máximos en primavera-verano (mayo-junio y julio-agosto, respectivamente), más tardíos a mayor altitud, por el efecto de la temperatura. En este sentido, detectamos un incremento generalizado del SAVI, un adelantamiento de la fase de reverdecimiento, culminado por un aumento del máximo reverdecimiento y seguido de una extensión de la estación de crecimiento. Se concluye que los ecosistemas de alta montaña en Andalucía muestran una tendencia estacional generalizada.
\end{abstract}

PALABRAS CLAVE: Oromediterráneo; crioromediterráneo; estaciones; fenología de la superficie terrestre; teledetección de la vegetación.

ABSTRACT: The high-mountain plant landscapes are a unique ecosystem in the southernmost region of the Iberian Peninsula, where the favourable season overlaps with a period of drought. In this work, we used the satellite images of the Terra-MODIS sensor to perform a seasonal trend analysis based on the Soil-Adjusted Vegetation Index (SAVI) 


\section{• O. GUTIÉRREZ-HERNÁNDEZ}

of 460 satellite images with a 16-day frequency, and applied to the surfaces covered by the plant communities of the oromediterranean and cryomediterranean bioclimatic levels in Andalusia, in order to provide the seasonal trend curves modelled (or fitted) for the first (2000) and last (2019) year of the time series. The results obtained allowed characterizing the typical phenological curve of these ecosystems, with minimums in winter (February) and maximums in spring-summer (May-June and July-August, respectively), later at higher altitudes, due to the effect of temperature. In this sense, we detected a general increase of the SAVI, an earlier start of the greening phase, that culminated by an increase in maximum greening and followed by an extension of the growing season. In conclusion, the high-mountain ecosystems in Andalusia show a general seasonal trend.

KEY WORDS: Oromediterranean; Cryomediterranean; seasons; land surface phenology; remote sensing of vegetation.

\section{Introducción}

Altitud, relieve, exposición e inclinación pueden llegar a producir en las montañas - de acuerdo con su impronta geográfica- modificaciones en las condiciones atmosféricas de su entorno. Así, la altitud lleva consigo un descenso general de la temperatura y un aumento de la radiación solar; y, el relieve condiciona la existencia de microambientes más o menos afectados por la radiación solar, el viento, la nieve o la humedad, debido a sus variantes de inclinación, exposición y configuración. Cuando las montañas tienen cierta entidad, producen una notable modificación de las características mesoclimáticas del entorno y condicionan el desarrollo de la vegetación (Lacoste \& Salonon, 1973).

Sólo determinadas especies han conseguido adaptarse a la alta montaña. Gran parte de la variación observada a lo largo de la elevación es el resultado de la sensibilidad fisiológica directa a las temperaturas y suministro de nutrientes (Laiolo \& Obeso, 2017). En este medio, los árboles están ausentes, las plantas anuales desaparecen y la vegetación está constituida básicamente por caméfitas, hemicriptófitas y geófitas (Ferreras \& Fidalgo, 1991). Aquí las formaciones vegetales se desarrollan con una reducción de sus partes aéreas y aparecen muy próximas al nivel del suelo, ocupando menos superficie, pero a través de una densificación del aparato vegetativo. Desde una perspectiva biogeográfica, el efecto del ascenso en altitud conlleva, en su escala, bastantes similitudes con el del incremento en latitud, y se traduce en una zonación altitudinal de la vegetación, esto es, el desarrollo de pisos de vegetación (Rubio, 1992).

España es uno de los países más montañosos de Europa, pero también es uno de los países en los que las montañas han sido más aprovechadas por las actividades humanas hasta el límite de lo posible, y esto es debido al predominio de la «montaña media» y a la escasa presencia — apenas $4.587 \mathrm{Km}^{2}$ - de la «alta montaña», si consideramos el umbral de esta más allá de los 2.000 metros de altitud, como señala Rodríguez (2001). Sin embargo, los umbrales entre la «montaña media» y la «alta montaña» dependen de los condicionantes geográficos -y en especial la latitud-, cuyos efectos extreman las condiciones mesoclimáticas del entorno y deter- minan la existencia de isleos microclimáticos de naturaleza orográfica que condicionan la existencia de comunidades vegetales muy especializadas o hasta la propia inexistencia de comunidad vegetal alguna en las cumbres más elevadas sometidas a la acción persistente del viento, el frío y la nieve (Webster, 1961).

Generalmente, en alta montaña ibérica, por debajo del nivel de las nieves perpetuas, hay un clima que durante las estaciones equinocciales podría ser equiparado con el que encontramos en la aciculisilva genuina, con la diferencia de que el verano mediterráneo impone excesos térmicos impensables en la taiga (Rubio, 1989). En efecto, en la cuenca mediterránea predomina un clima que, aunque diverso y a menudo extremo en matices, se distingue por un pronunciado déficit pluviométrico en los meses centrales del verano coincidiendo con el momento en el que las temperaturas son más elevadas, una singularidad propia de los climas subtropicales en los que reinan los veranos secos por el desplazamiento hacia latitudes medias de la subsidencia subtropical durante el estío (Gil \& Olcina, 2017). En las montañas del sur de la península ibérica, los rigores del clima se manifiestan tanto en los inviernos, por el frío, el viento, las heladas y las nieves; como en los veranos, porque en los segundos, en los que se impone una intensa radiación, además, la sequía estival es tan acusada como en el resto de la región, y ni siquiera el efecto orográfico de las máximas elevaciones puede anteponerse a la marcada subsidencia del aire procedente de las altas presiones subtropicales (Pita, 2003). Sin embargo, y pese a la sequía, es en este momento cuando en la alta montaña mediterránea, con la fusión de los hielos, con unas temperaturas más suaves que en las zonas circundantes y con la presencia de cierta humedad en el sustrato edáfico, se encuentra el ambiente más propicio para las comunidades adaptadas a la dureza de este clima, como ocurre en los borreguiles de Sierra Nevada (Pérez-Luque et al., 2015).

En Andalucía, la Consejería de Medio Ambiente de la Junta de Andalucía considera ecosistemas de alta montaña aquellos situados por encima de los $1.700 \mathrm{~m}$ de altitud en los que se produce «la aparición de condiciones climáticas muy duras en relación con la temperatura, la precipitación, la radiación solar y el viento, y la permanencia de 
la nieve durante gran parte el año, lo que limita la estación de crecimiento de la vegetación hasta la desaparición de los árboles, y solo perviven las especies con adaptaciones especiales» (Cabello \& Castro, 2012). En lo que se ha venido en denominar «Alta Montaña Bética», la aparición del piso bioclimático oromediterráneo coincide con el límite superior de los frondosos bosques del piso supramediterráneo. En el oromediterráneo, la vegetación potencial suele ser un bosque muy abierto de coníferas y con un sotobosque en el que predomina un matorral de sabinas, enebros rastreros y piornos almohadillados (Valle \& Navarro, 2004), un ámbito caracterizado por la existencia de un marcado contraste estacional.

La fenología estudia los eventos periódicos del ciclo de vida de las plantas y los animales y cómo estos están influenciados por las variaciones estacionales e interanuales en el clima, así como por los factores del hábitat (Schwartz, 2013). La fenología de la superficie terrestre (LSP, de Land Surface Phenology) consiste en el estudio multiescalar de la fenología de la vegetación de acuerdo con los datos adquiridos mediante el uso de sensores ópticos espaciales (Helman, 2018). Las ventajas de utilizar la teledetección para aplicaciones de fenología radican en la capacidad de capturar la expresión continua de patrones fenológicos en todo el paisaje y la capacidad de observar retrospectivamente la fenología de los conjuntos de datos satelitales almacenados en bases de datos espaciales (Reed et al., 2009).

Aunque la caracterización espectral de la vegetación constituye una de las tareas más recurrentes en los trabajos de teledetección (Jones \& Vaughan, 2010), todavía ofrece notables dificultades como consecuencia de los múltiples factores que inciden en la radiancia que detecta un sensor remoto, entre los cuales destacan: los relacionados con la reflectividad de las hojas; con las características geométricas de las plantas; y con las características del entorno en el que se desarrollan las comunidades vegetales (Chuvieco, 2008). El empleo de cocientes o índices de vegetación para discriminar entre las cubiertas vegetales y entre estas y otras cubiertas, se deriva del peculiar comportamiento espectral de la vegetación, que ofrece un contraste máximo entre las bandas del espectro visible y las bandas del infrarrojo (Myneni et al., 1995). El denominado Índice de Vegetación de Diferencia Normalizada (NDVI, del inglés Normalized Difference Vegetation Index), propuesto en 1974 (Rouse et al., 1974), se encuentra entre los más empleados. Sin embargo, entre los factores que modifican sustancialmente el comportamiento del NVDI figura la proporción vegetación / suelo (Gilabert et al., 2002), que es precisamente uno de los mayores problemas que encontramos en el estudio de las comunidades vegetales donde asoma con frecuencia el suelo cuya señal también es recibida por los sensores. Para solucionar este problema e incluir explícitamente el factor suelo, Huete (1988) propuso el Índice de Vegetación Ajustado al Suelo, el SAVI (Soil Adjusted Vegetation Index), un índice que posteriormente ha demostrado mejorar las prestaciones del NDVI en formaciones vegetales arbustivas y herbáceas donde la presencia del suelo introduce notables cantidades de ruido en la señal espectral detectada por los sensores remotos (Huete et al., 1992).

En este trabajo, se usaron las imágenes de satélite del sensor Terra-MODIS para desarrollar un análisis de tendencia estacional basado en el cálculo del Índice de Vegetación Ajustado al Suelo (SAVI) de imágenes con frecuencia de 16 días, un análisis sobre las superficies cubiertas por las comunidades vegetales de los pisos oromediterráneo y crioromediterráneo en Andalucía, con el fin obtener las curvas de tenencia estacional modeladas (o ajustadas) para el primer (2000) y último (2019) año de la serie. Se consideran dos hipótesis de investigación:

1. Primera, el Índice de Vegetación Ajustado al Suelo (SAVI) es apropiado para el estudio de superficies con vegetación abierta y puede ser sensible a la percepción de los ciclos fenológicos en estas comunidades vegetales típicas de los ecosistemas de alta montaña.

2. Segunda, en un contexto de cambio global caracterizado esencialmente por el incremento general de las temperaturas, se estima encontrar una tendencia estacional de estas comunidades vegetales adaptadas al microclima de montaña.

\section{Material y métodos}

\section{1. Área de estudio}

Antiguamente, se acostumbrada a diferenciar en Andalucía dos grandes conjuntos subregionales contrastados: Andalucía Occidental (Huelva, Cádiz, Sevilla y Córdoba) y Andalucía Oriental (Málaga, Granada, Jaén y Almería). Aunque hoy persiste el uso de esta diferenciación, normalmente esta suele emplearse para hacer referencia a los aspectos generales del medio físico, para así contraponer la Alta Andalucía frente a la Baja Andalucía (López, 2003). En efecto, la «Alta Montaña Bética» se localiza en las provincias orientales andaluzas: Almería, Granada, Jaén y Málaga, aunque en esta última provincia aparece en un área muy reducida. En este trabajo, estudiamos los ecosistemas de alta montaña situados por encima de los $1.700 \mathrm{~m}$ de altitud, de acuerdo con el criterio adoptado por la propia Consejería de Medio Ambiente de la Junta de Andalucía, y sólo allí donde se encuentran comunidades vegetales de los pisos oromediterráneo y crioromediterráneo (Figura 1).

De acuerdo con la caracterización climática de Andalucía a escala regional de Gómez-Zotano et al. (2015), estas áreas se hayan clasificadas bajo la denominación de «Clima de alta montaña». Se trata de un clima frío o muy frío - en el caso de las altas cumbres de Sierra Nevada-, y de carácter seco a subhúmedo (localmente húmedo), en los que predomina una elevada continentalidad. Se encuentran dos pisos bioclimáticos de alta montaña (RivasMartinez, 1987): el piso oromediterráneo entre los 1.700- 


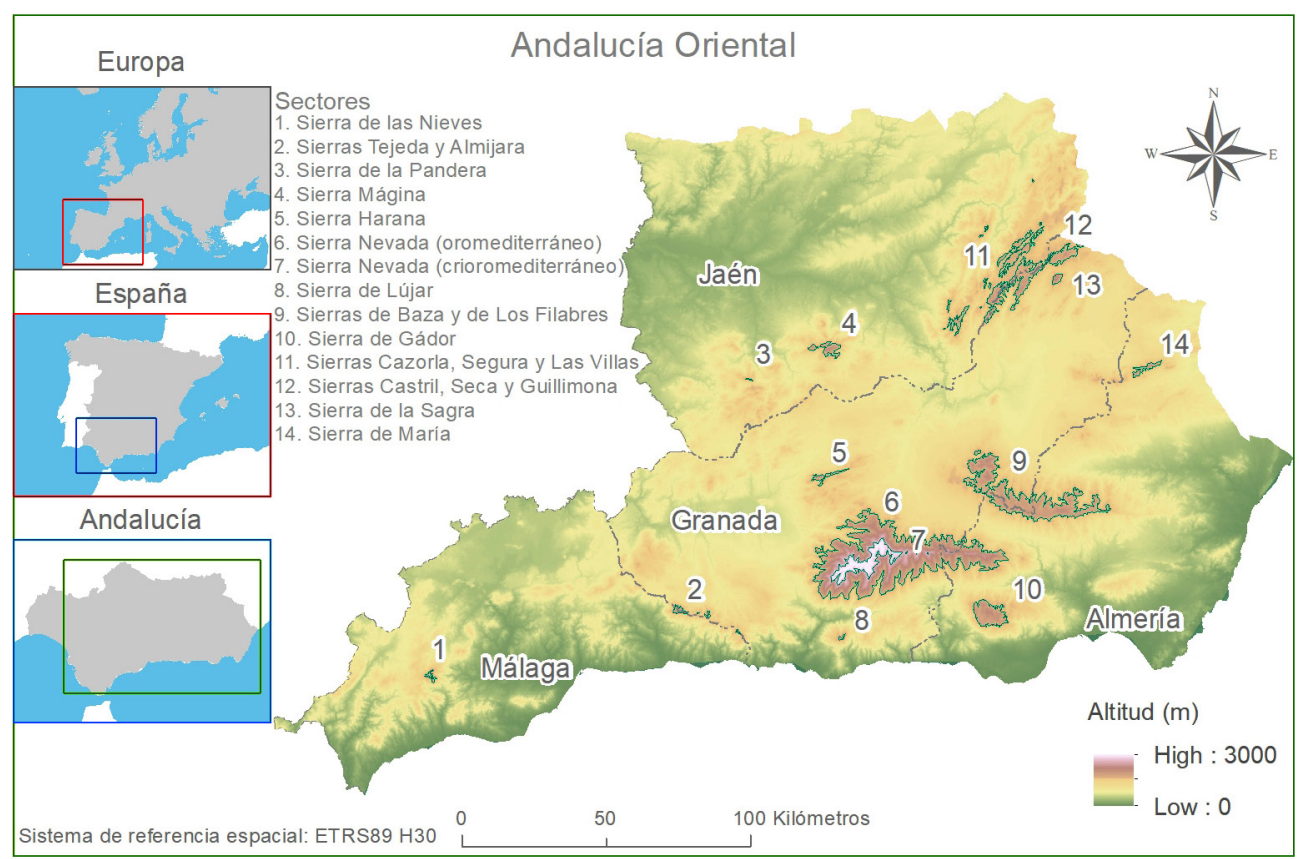

Figura 1: Área de estudio. Fuente: Elaboración propia. A partir de DERA-IECA y REDIAM. Figure 1: Study area. Source: own elaboration. Based on DERA-IECA \& REDIAM.

$1800 \mathrm{~m}$ y los $2.700 \mathrm{~m}$, aproximadamente, y el piso el crioromediterráneo por encima de los $2.700 \mathrm{~m}$, aunque estos umbrales varían en función de la exposición del relieve. En el piso oromediterráneo, la temperatura media anual oscila entre $\operatorname{los} 8$ y $\operatorname{los} 4^{\circ} \mathrm{C}$, con temperaturas medias de las mínimas del mes más frío entre -1 y los $-4^{\circ} \mathrm{C}$; $\mathrm{y}$, en el piso crioromediterráneo, la temperatura media anual baja de $\operatorname{los} 4^{\circ} \mathrm{C}$ y la temperatura media de las mínimas del mes más frío es inferior a $-7^{\circ} \mathrm{C}$. Por tanto, los inviernos son rigurosos, con heladas recurrentes y temperaturas negativas durante varios meses. La nieve es un meteoro frecuente, aunque sólo es persistente durante la mayor parte del año en las altas cumbres de Sierra Nevada. El verano puede ser considerado como una estación templada con valores máximos absolutos superiores a $25^{\circ} \mathrm{C}$ en las cotas más bajas y expuestas al mediodía del piso oromediterráneo. Desde el punto de vista pluviométrico, este clima de alta montaña, como el resto de la región, presenta un gradiente decreciente oeste a este y de noroeste a sureste, con precipitaciones que van desde los $1500 \mathrm{~mm}$ en las zonas de barlovento con respecto a los flujos húmedos de poniente frente a los $500 \mathrm{~mm}$. Tiene un máximo otoño-invernal, que se desplaza hacia la primavera en las montañas del noreste. Una prolongada sequía y una intensa insolación condicionan la estación estival y determinan una acusada xericidad del paisaje vegetal.

La superficie muestreada incluye catorce sectores montañosos que suman una superficie de 80.763 hectáreas, trece de los cuales incluyen comunidades vegetales del piso oromediterráneo, situadas en su mayor parte entre los 1.700 y los $1.900 \mathrm{~m}$ de altitud media, con una superficie de 70.143 hectáreas. El piso crioromediterráneo sólo aparece en las cumbres de Sierra Nevada, por encima de los 2.700 m, sobre una superficie de 10.620 hectáreas (Figura 2).

Desde el punto de vista biogeográfico, estas montañas se encuentran en la región Mediterránea, subregión Mediterránea occidental, superprovincia Mediterráneo-Iberoatlántica, y en la provincia Bética (Rivas-Martinez, 1987). El paisaje vegetal de la alta montaña andaluza es muy característico (Figura 3). En el piso oromediterráneo abundan las formaciones arbustivas de sabinas y enebros, distinguiéndose las comunidades basófilas sobre sustratos calcáreos y comunidades basófilas sobre sustratos silíceos; y, en el piso crioromediterráneo sólo se encuentran pastizales de alta montaña (Ibarra, 2003). Las formaciones arboladas que se estima en el pasado fueron más frecuentes, actualmente son escasas y abiertas, excepto en Sierra Nevada y alineación Baza-Los Filabres, donde se mantienen importantes bosques de pino silvestre procedentes de antiguas repoblaciones (Mesa, 2016, 2020; Olmedo, 2012).

\subsection{Metodología}

Esta es la secuencia metodológica llevaba a cabo en la presente investigación:

1. Sectorización del área de estudio. La delimitación de los pisos oromediterráneo y crio oromediterráneo se elaboró en formato vectorial por medio de un Sistema de Información Geográfica y partiendo de la cartografía de referencia elaborada por la Consejería de Medio Ambiente de la Junta de Andalucía en formato vectorial, Distribución de los 
FENOLOGÍA DE LOS ECOSISTEMAS DE ALTA MONTAÑA EN ANDALUCÍA: ANÁLISIS DE LA TENDENCIA... • 5

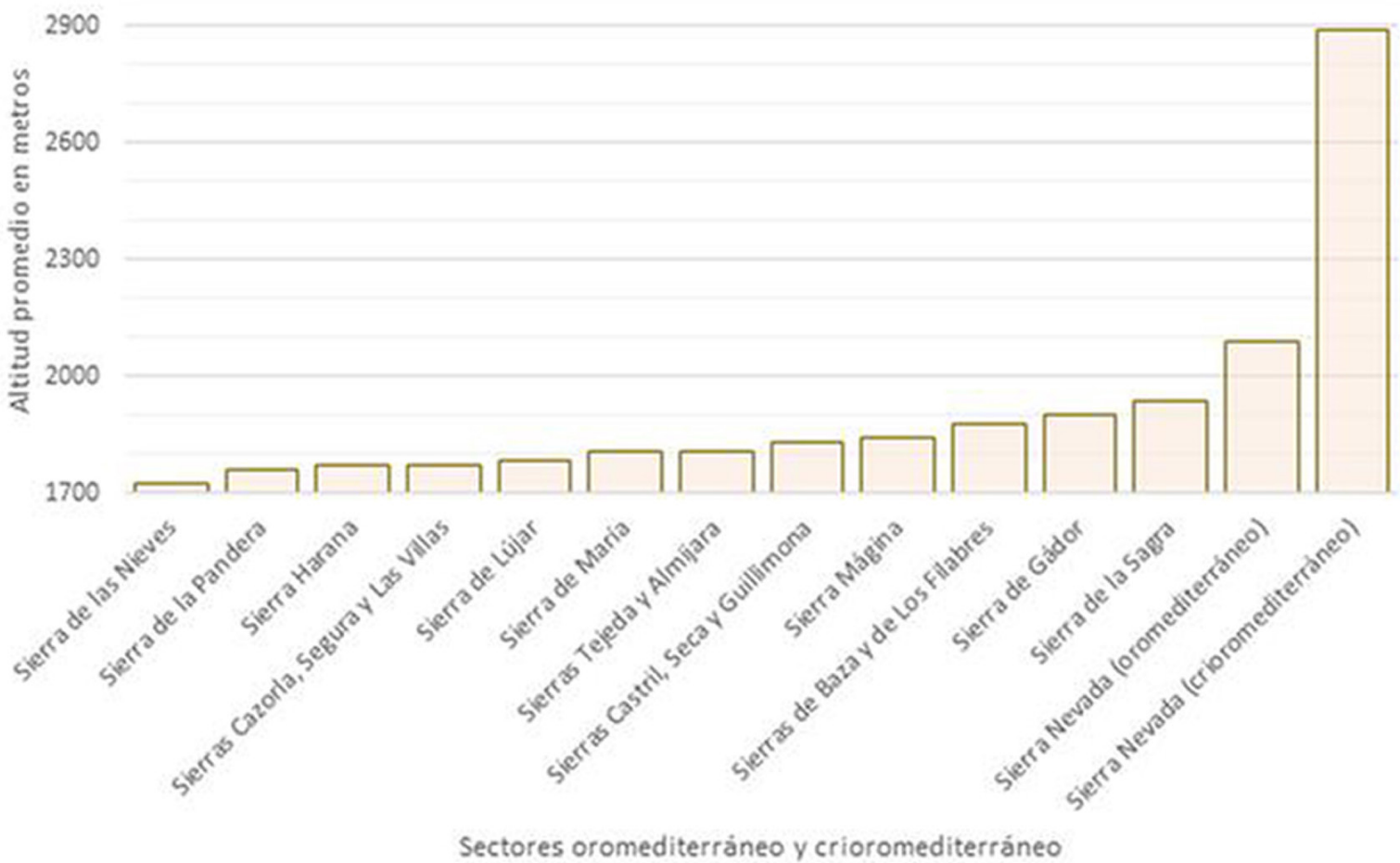

Figura 2: Altitud media de los sectores montañosos estudiados: Elaboración propia. A partir de DERA-IECA. Figure 2: Study area. Source: own elaboration. Based on DERA-IECA.

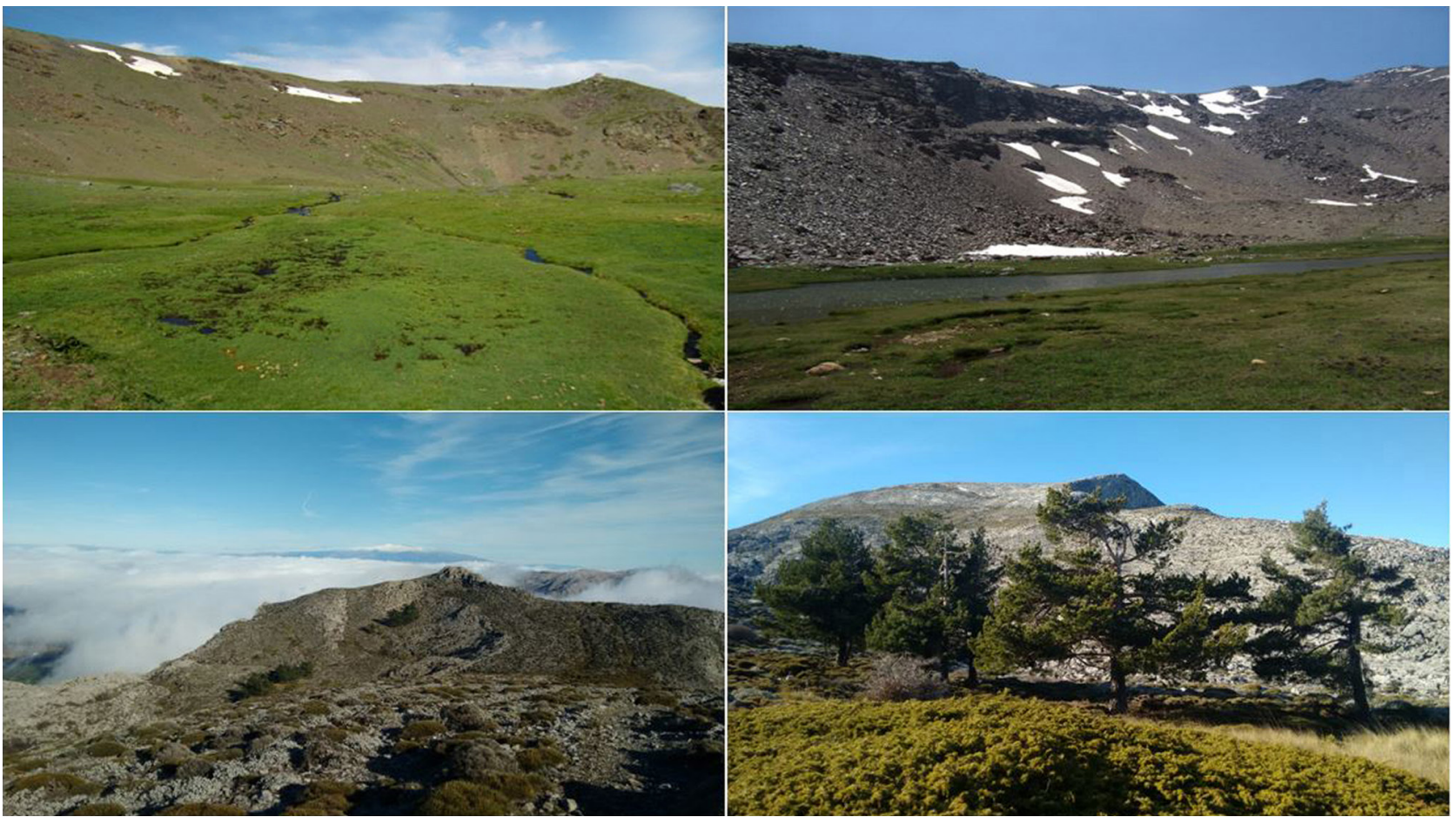

Figura 3: Formaciones herbáceas silícolas en los pisos oro y crioromediterráneo de Sierra Nevada (Arriba). Formaciones arbustivas calcícolas y pinares en el piso oromediterráneo de Sierra Tejeda (Abajo). Fuente: Elaboración propia.

Figure 3: Siliceous herbaceous formations in the cryo-oromediterranean level in Sierra Nevada (Above). Calcareous shrub formations and pine forests in the oromediterranean level in Sierra Tejeda (Below). Source: own elaboration. 
pisos bioclimáticos a partir de la cartografía de vegetación 1.10.000. Se generó una nueva versión adaptada basada en los criterios especificados en el apartado anterior, y en la que sólo se incluyeron aquellos sectores con un área mínima de suficiente entidad como para registrar una señal genuina de los pisos bioclimáticos de alta montaña.

2. Descarga de imágenes de satélite. Se trabajó con las imágenes del sensor Terra-MODIS, en concreto, el producto MOD13Q1, donde cada imagen es obtenida a partir de la selección de los píxeles aceptables de un compuesto de 16 días, lo que incluye un total de 23 imágenes anuales con una resolución espacial de 250 metros (Didan, 2015; Mas, 2011). Inicialmente, se descargaron las imágenes correspondientes con las bandas 1 (rojo) y 2 (infrarrojo) de las series anuales completas del producto MOD13Q1 desde los años 2000 a 2019, exceptuando la primera imagen del año 2000, ya que no está disponible, dato faltante que se generó posteriormente (véase paso 4. Procesamiento de la serie temporal).

3. Cálculo del índice de vegetación SAVI. Dado que en este trabajo se analizaron superficies cubiertas en su mayor parte por formaciones arbustivas y abiertas, con presencia abundante de suelo y roquedo, se calculó el Índice de Vegetación Ajustado al Suelo o SAVI (Soil Adjusted Vegetation Index), propuesto por Huete (1988), y mejorado por éste y colaboradores (Huete et al., 1992), y que, a diferencia del NDVI, el SAVI está más estrechamente relacionado con el índice de área foliar en el rango de reflectancias del suelo (Baret et al., 1993; Jones \& Vaughan, 2010).

$$
S A V I=\frac{\rho(I R C-\rho R)}{\rho(I R C+\rho R+L)}(1+L)
$$

donde: $\rho I R C$ es reflectividad en el canal infrarrojo; $\rho R$ es reflectividad en canal rojo; $L$ es una constante para ajustar la línea vegetación-suelo al origen: se ha ajustado en torno a un valor de 0,5 , una solución intermedia de acuerdo a Huete et al., (1994), Chuvieco (2008) y Jones \& Vaughan (2010).

4. Procesamiento de la serie temporal. Con el fin de obtener una serie temporal completa, uniforme y coherente, válida para todo tipo de análisis estadísticos: 1) Se interpolaron los valores faltantes correspondientes a enero de 2000 a través del panel Missing Data Interpolation del módulo Earth Trend Modeller de Terrset, ya que las primeras imágenes disponibles del sensor MODIS son de febrero del año 2000; 2) Se corrigieron los píxeles con valores erróneos a través de una adaptación del procedimiento de HANTS - Harmonic Analysis of Time Series (Roerink et al., 2000).

5. Análisis de datos y modelización. Se desarrolló un análisis de tendencia estacional basado en las 460 imágenes con el SAVI obtenidas previamente con la metodología de Eastman et al. (2009, 2013). Este método calcula una regresión armónica de imágenes anuales seguida de un análisis de la amplitud y las fases de cada año generadas por la regresión armónica. La regresión armónica es similar al análisis de Fourier, pero permite la especificación de fechas julianas. Se utilizan dos o cuatro frecuencias para la regresión armónica con el fin de capturar curvas estacionales y tendencias dominantes en los datos sin incluir el ruido de alta frecuencia. De este modo, se modeló la curva estacional para cada periodo de referencia y se estimaron los parámetros fenológicos básicos derivados de los periodos de reverdecimiento o Green up (Green up onset y Green up phase) y senescencia o Green down (Green down onset y Green down phase) (Zhang et al., 2003, 2012). Para el análisis de la fase del Green up y la fase del Green down, el umbral de la curva se estableció en el punto en que la cantidad de verde excede el $40 \%$ de toda la trayectoria desde el valor mínimo hasta el máximo.

6. Representación de curvas estacionales modeladas (o ajustadas). Los resultados obtenidos en esta investigación representan, a partir de la serie temporal de imágenes de satélite con frecuencia de 16 días del SAVI que abarca 20 años, las curvas estacionales modeladas - y sus parámetros asociados - para los años $2000\left(t_{1}\right)$ y $2019\left(t_{2}\right)$ en el interior de cada una de las áreas de estudio muestreadas (véase figuras 1 y 2). Es importante tener en cuenta que se trata de curvas ajustadas, esto es, similares a una línea de tendencia de regresión que en este caso se deriva del análisis de toda la serie temporal del SAVI con frecuencia de 16 días. Es decir, estas curvas no son simples descripciones de lo que se registra en los años 2000 y 2019 específicamente, sino que son las curvas que mejor se ajustan a 2000 y 2019 considerando todos los datos de la serie temporal. Esta visión intencionalmente generalizada se basa en la mayor cantidad posible de información y es una abstracción que rechaza intencionalmente la variabilidad a corto plazo.

La descarga de imágenes de satélite y el cálculo del SAVI se llevó a cabo en el entorno computacional de análisis de datos R (R Core Team, 2019) a través del paquete MODIStsp (Busetto \& Ranghetti, 2016). El pre-procesamiento de la serie temporal de imágenes de satélite se hizo con el software Terrset, y utilizamos su módulo Earth Trend Modeller para desarrollar el análisis estacional (Eastman, 2015). El Sistema de Información Geográfica empleado fue ArcGIS 10.7, con el que digitalizamos los sectores del área de estudio, integrados los datos espaciales y producimos la cartografía de referencia.

\section{Resultados}

Los resultados se muestran en las figuras 4 y 5 , donde se representan las curvas fenológicas modeladas del 

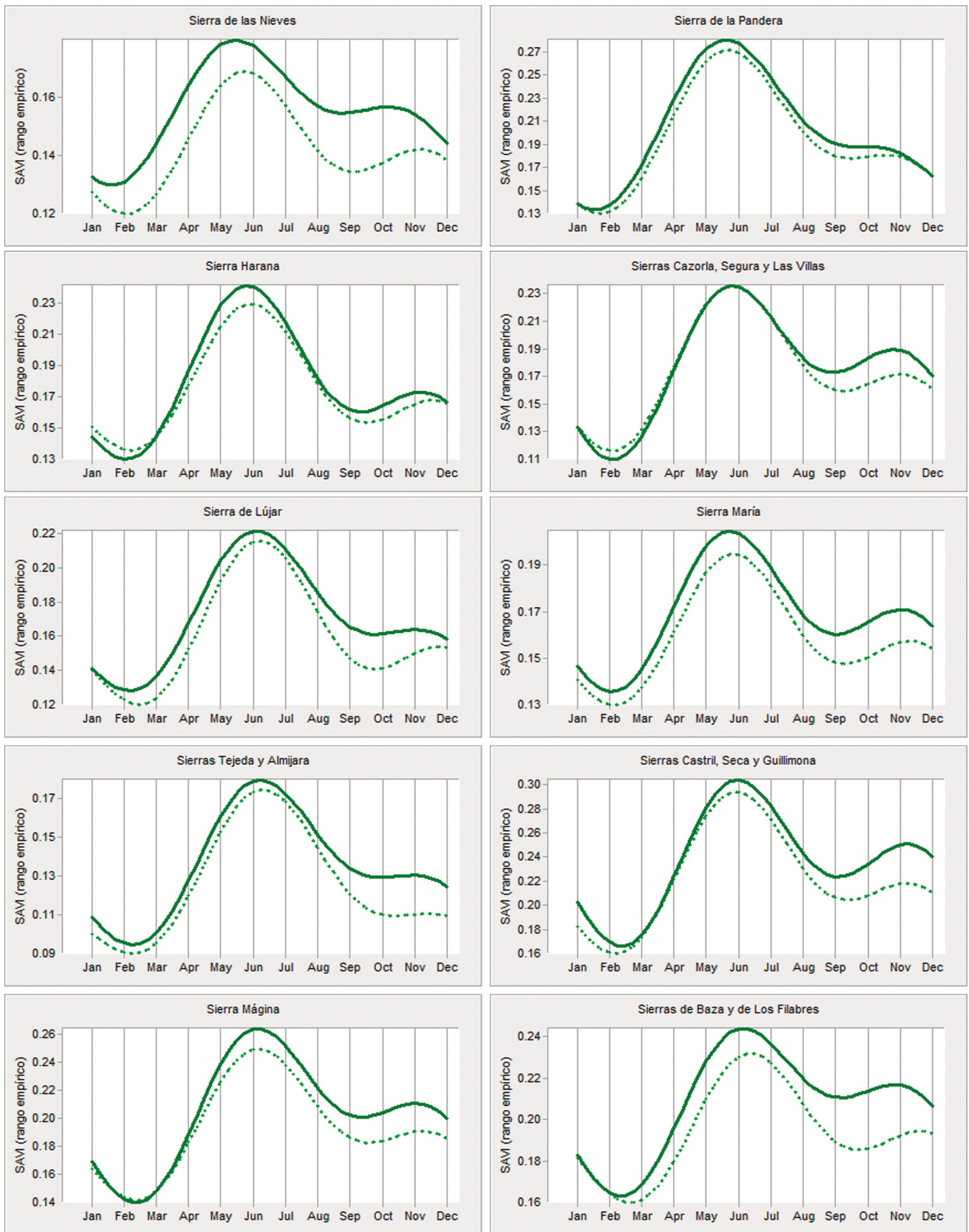

Figura 4: Curvas estacionales del SAVI en sectores montañosos oromediterráneos ordenados de menor a mayor altitud media. Línea discontinua: 2000; Línea continua: 2019. Fuente: Elaboración propia.

Figure 4: Seasonal curves of the SAVI in the in the mountainous oromediterranean areas ordered from lowest to highest average altitude. Dashed line: 2000; Continuous line: 2019. Source: own elaboration. 

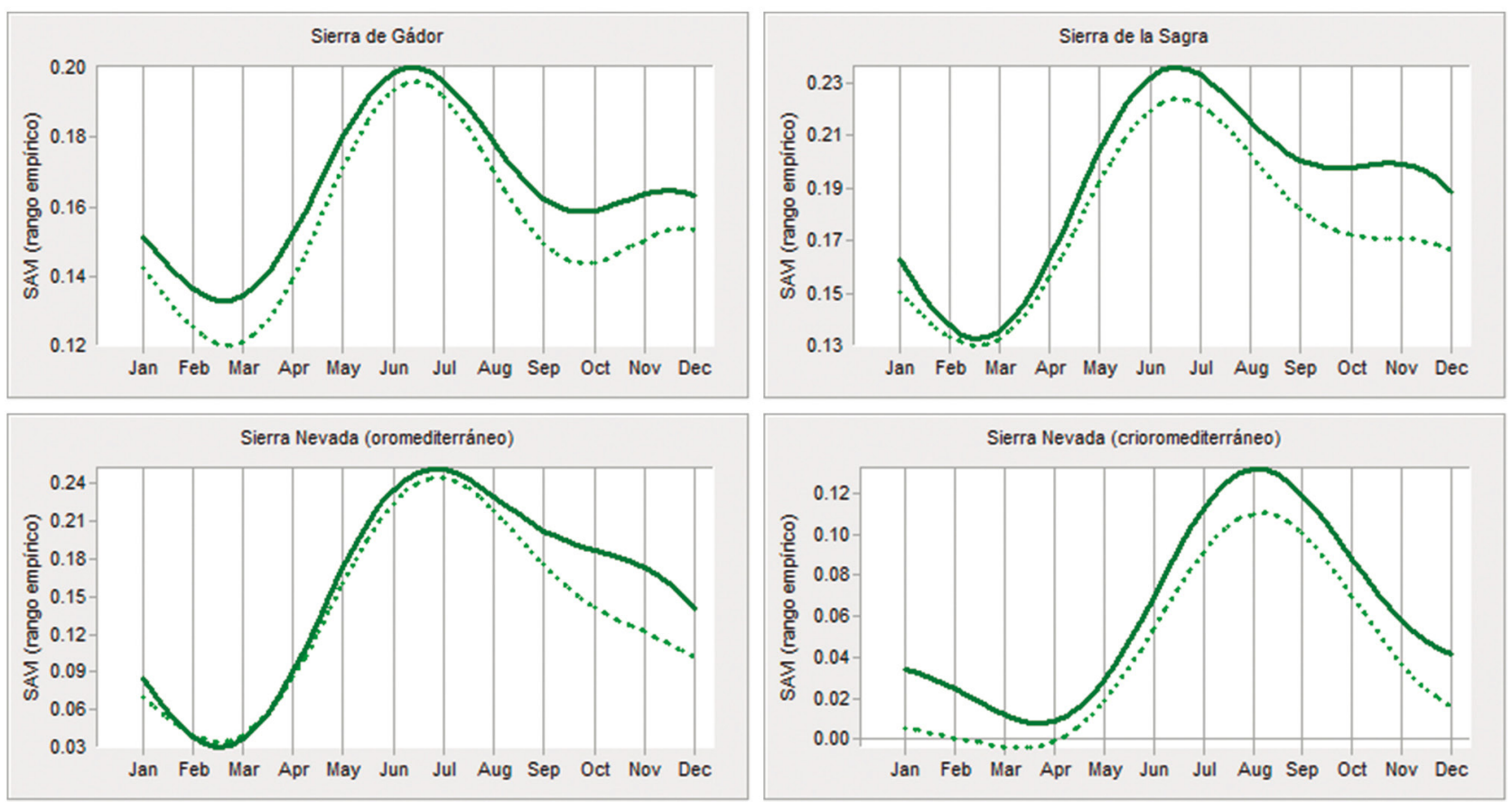

Figura 5: Curvas estacionales del SAVI en sectores montañosos oromediterráneos y crioromediterráneo ordenados de menor a mayor altitud media. Línea discontinua: 2000; Línea continua: 2019. Fuente: Elaboración propia.

Figure 5: Seasonal curves in the mountainous oromediterranean and cryo-oromediterranean areas ordered from lowest to highest average altitude. Dashed line: 2000; Continuous line: 2019. Source: own elaboration.

SAVI para los años 2000 y 2019 en los distintos sectores muestreados en Andalucía de los pisos oromediterráneo y crioromediterráneo. A nivel general, el valor promedio del SAVI fue de 0,18 $(\sigma=0,03)$; el valor mínimo de $0 ; \mathrm{y}$, el valor máximo de 0,30 . Se detectó un aumento generalizado del SAVI consistente en casi todos los sectores muestreados.

Como se ha adelantado, el valor mínimo del SAVI se produce durante el invierno y, en la mayor parte de los sectores, este mínimo se sitúa por encima de 0,1 - exceptuando Sierra Nevada. El mínimo más frecuente se produce en torno al mes de febrero, aunque se encontró desde mediados de enero-febrero, en las sierras con menor altitud, como Sierra de las Nieves o Sierra de la Pandera; hasta febrero-marzo, en la mayor parte de los sectores estudiados, e incluso un poco más tarde, marzo-abril, en el piso crioromediterráneo de Sierra Nevada. Considerando el conjunto de los sectores, los valores mínimos encontrados en 2019 fueron ligeramente superiores a los encontrados en 2000, aunque con escasas diferencias y varias excepciones, caso de Sierra Harana o las Sierras Cazorla, Segura y Las Villas. Por el contrario, el valor máximo del SAVI se produce entre primavera y verano, desde los meses de mayo-junio, en las sierras menos elevadas, hasta el mes de agosto, en las cumbres de Sierra Nevada. Nuevamente, se encontró un retraso de los valores máximos de acuerdo con la altitud promedio de cada sector: en general, a mayor altitud, el máximo del SAVI se alcanza más avanzado el verano.

Los máximos y mínimos de las curvas estacionales proyectan -especialmente en el piso oromediterrá- neo- una onda asimétrica con un mínimo principal en enero-febrero y un mínimo secundario en septiembreoctubre, aunque en muchos sectores el SAVI se mantiene e incluso repunta en noviembre, originando un máximo secundario, aunque de una menor entidad. Si anteriormente se indicaba la existencia de un aumento general del SAVI, también se detectó un aumento del SAVI consistente en todos los meses y en casi todos los sectores, por lo que se mantiene la misma forma en las curvas modeladas para 2000 y 2019 , aunque durante los meses de otoño este aumento es mayor y se produce consecuentemente una modificación de la forma curva de 2019 durante esta estación.

De acuerdo con las curvas modeladas del SAVI para 2000 y 2019 , se detectó un adelantamiento —en torno a 6 días- del periodo de reverdecimiento (Green up) en casi todos los sectores, máximo en la Sierra de las Nieves y mínimo en las sierras del noreste de la región: Sierras Cazorla, Segura y Las Villas, y Sierras Castril, Seca y Guillimona. El inicio del Green up se produce entre marzo y abril en el piso oromediterráneo, y en mayo en el piso crioromediterráneo. El valor máximo del SAVI, en el cual también se detectó un consiguiente adelantamiento, se halló a finales de mayo en las sierras de menor altitud, y a comienzos de agosto en las cumbres más elevadas de Sierra Nevada. Por último, se observó un alargamiento generalizado de la estación de crecimiento por adeltanto del Green up y extensión del Green brown phase, que en algunos sectores se prolonga hasta finales de la estación otoñal. 


\section{Discusión}

Globalmente, las curvas obtenidas con el SAVI plasman el típico comportamiento estacional de la vegetación de alta montaña mediterránea, con valores mínimos y máximos relativamente contrastados en invierno y primavera-verano respectivamente, y con una pendiente muy acentuada hacia las estaciones equinocciales, especialmente durante el tránsito del invierno hacia primavera-verano; Todo lo contrario de lo que ocurre en los ecosistemas mediterráneos dominados por formaciones arbustivas y herbáceas y situados en pisos bioclimáticos basales, con curvas fenológicas que presentan unos valores mínimos durante el verano, coincidiendo con el periodo de máximo extrés por sequía (Alcaraz-Segura et al., 2009; Alcaraz-Segura, 2006; Bergmeier, 1998). En este sentido, el SAVI ha demostrado una gran sensibilidad para captar tanto las variaciones estacionales como sus tendencias en un entorno montañoso donde predominan formaciones arbustivas y herbáceas y en el que el «ruído del suelo» (nieve, roquedo) introduce notables modificaciones en la «señal de la vegetación», por lo que este índice cumple en nuestro entorno el fin para el que fue concebido y desarrollado en origen (Huete, 1988; Huete et al., 1992).

Los valores mínimos del SAVI se relacionan tanto con una menor actividad vegetal vinculada con unas temperaturas más bajas y debido a la presencia de la cobertura nival durante el invierno y parte de las estaciones equinocciales, especialmente en las sierras más elevadas, donde destaca Sierra Nevada sobre el resto. El mínimo principal se produce avanzado el invierno en los meses fríos en los que la cobertura de nieve es más frecuente; y, el mínimo secundario se registra en relación con los efectos acumulados de la sequía estival, que remiten con las lluvias otoñales, más frecuentes entre noviembre y diciembre (Pita, 2003). Por el contrario, los valores máximos registrados se relacionan con la reducción y desaparición de la cobertura nival, y con un aumento de la temperatura en el contexto de un ambiente subhúmedo y húmedo en la alta montaña mediterránea, que origina un aumento de la actividad vegetal. En efecto, se ha demostrado que la temperatura es el principal impulsor de muchos procesos de desarrollo de la planta, y en muchos casos se ha demostrado que temperaturas más altas aceleran el desarrollo de las comunidades vegetales (Vandvik et al., 2018; Zhang, 2012). Esto es coherente con el hecho de que en nuestro estudio se encontraron gradientes fenológicos en función de la altitud promedio de cada sector, con diferencias que podrían estar explicadas por la existencia de diversas formaciones vegetales y distintas condiciones de humedad.

Diferentes autores han puesto de manifiesto un adelantamiento del periodo de reverdecimiento y una extensión del periodo de senescencia, circunstancia que otros autores han vinculado con un aumento de la temperatura (Badeck et al., 2004; Ma et al., 2016; Wang et al., 2015). En general, se ha mantenido la forma de las curvas estacionales, y lo que ha cambiado es el SAVI promedio, he- cho que produce que la curva de 2019 se sitúe por encima de la curva de 2000; estos mismos cambios han sido descritos por Eastman et al. (2009), y suelen estar originados por un aumento consistente de la temperatura en todos los meses del año, lo que ocasiona en consecuencia un adelanto de la fase de reverdecimiento y un retraso de la fase de senescencia por extensión de la estación de crecimiento. En efecto, el adelanto del Green up es interpretado como un efecto del aumento de la temperatura (Richardson et al., 2013) aunque también encontramos una extensión de la estación de crecimiento, lo que se relaciona con un consiguiente aumento del secuestro de carbono (White et al., 2009), pero también con una mayor exposición de esta vegetación de alta montaña a un posible estrés por la sequía (Zhang et al., 2009).

Este reverdecimiento, sin embargo, podría estar operando simultáneamente junto con un ensamblaje de efectos negativos sobre especies endémicas y especialistas de la alta montaña mediterránea (Rixen \& Wipf, 2017). Diferentes investigaciones han evidenciado que el calentamiento global está afectando a los hábitats de alta montaña (Dullinger et al., 2012; Engler et al., 2011; Fort, 2015), que reducirán su área de distribución debido al aumento de migración de especies de zonas bajas. De hecho, este incremento generalizado del SAVI podría ser la antesala de una migración altitudinal de especies procedentes de pisos bioclimáticos inferiores. De confirmarse este proceso, estaríamos ante una reducción de la diversidad de microhábitats. De hecho, podría aflorar un problema crítico en ecosistemas tan vulnerables al incremento de la sequía como la alta montaña mediterránea, donde la estación de crecimiento coincide con el periodo de sequía, pues los cambios previstos en el clima llevarán a una situación de déficit hídrico durante los períodos de crecimiento de la vegetación (Catalan et al., 2017). En este punto, conviene recordar que las montañas contribuyen desproporcionadamente a la biodiversidad terrestre de la Tierra, pero el papel de estas como refugios para la biodiversidad podría verse amenazado por el cambio climático (Rahbek et al., 2019).

Un aspecto que convendría remarcar en cuanto a una posible explicación de las diferencias encontradas en los diferentes sectores y cuyas causas exceden los objetivos de esta investigación, se relaciona con las singularidades propias de cada sector. Es necesario conocer en profundidad las interacciones que se producen localmente entre el clima, procesos ecológicos y actividades humanas en el pasado para comprender mejor el comportamiento de los ecosistemas en el presente y el futuro. En este sentido, la actividad humana no se puede desacoplar del paisaje, porque de hecho explica buena parte de la resiliencia encontrada en determinados ecosistemas de montaña frente al cambio global (Huber et al., 2005). Así, el conocimiento acerca de la historia de los usos del suelo en los regiones montañosas también es decisivo para explicar las respuestas de un ecosistema de montaña ante el cambio global a partir de los condicionantes encontrados en las trayectorias históricas de sus formaciones vegetales (Pèlachs et al., 2017). Y finalmente se ha de tener en 
cuenta que la montaña integra una gran heterogeneidad ambiental y espacial que se manifiesta en las unidades geomorfoedáficas y los matices bioclimáticos que conjuntamente proyectan un complejo mosaico de unidades de vegetación que pueden experimentar respuestas contrastadas ante el cambio global (Shafer et al., 2005).

Por último, en el capítulo de limitaciones de los resultados obtenidos y futuras líneas de investigación encaminadas a ampliar sus horizontes, aunque se considera que la resolución temporal es más que suficiente para la modelización de las curvas estacionales, y que a escala de sector la resolución de 250 metros produce resultados aceptables, esta resolución espacial podría resultar insuficiente para el conocimiento detallado de la respuesta fenológica en comunidades o formaciones vegetales específicas. En este sentido, el trabajo con índices de vegetación ajustados al suelo con un rango dinámico de $L$ podría introducir mejoras en aspectos relacionados con la exactitud y precisión de la señal recibida (Qi et al., 1994), sin cambiar la resolución de los datos ni la fuente de registro, pero con la necesidad de realizar trabajos de campo que permitan calibrar empíricamente estos modelos. Por el contrario, si prescindimos de un ajuste del rango dinámico de $L$, podríamos obtener una señal de la vegetación más genuina a escala de detalle siempre y cuando trabajásemos con sensores de mayor resolución como los disponibles en los satélites de la series Landsat y Sentinel.

\section{Conclusiones}

En este trabajo, se ha llevado a cabo un análisis estacional del Índice de Vegetación Ajustado al Suelo (SAVI), basado en una serie temporal de imágenes satelitales Terra-MODIS con una frecuencia de dieciséis días desde enero de 2000 hasta diciembre de 2019, con la modelización de las respectivas curvas estacionales para el primer y último año de la serie por medio de un análisis estacional elaborado con regresiones armónicas (similares a un análisis de Fourier). En relación con los objetivos e hipótesis que se plantearon inicialmente, concluimos que:

- El SAVI permite capturar la señal de la vegetación arbustiva y herbácea en ecosistemas de alta montaña de los pisos oromediterráneo y crioromediterráneo en los que la reflectancia que reciben los sensores remotos está influenciada por el ruido de otras coberturas no vegetales del suelo. La modelización de curvas estacionales basadas en las series temporales del SAVI registra las curvas fenológicas características de la vegetación de montaña y la variabilidad estacional e interanual.

- Las curvas de tendencia estacional modeladas (o ajustadas) representan la señal fenológica característica de los ecosistemas de alta montaña mediterráneos, con valores mínimos en invierno (febrero) y máximos en primavera-verano (mayo-junio y julioagosto), más tardíos a mayor altitud; y proyectan una onda asimétrica con una pendiente más pronunciada en la fase de reverdecimiento que en el periodo de senescencia. En este sentido, entre las curvas modeladas para el año 2000 y el año 2019, detectamos un incremento generalizado del SAVI, un adelantamiento de la fase de reverdecimiento, culminado por un aumento del máximo reverdecimiento y seguido de una extensión de la estación de crecimiento.

Los resultados sugieren que la temperatura juega un rol fundamental en la dinámica estacional de la vegetación en los ecosistemas de alta montaña del sur de la península ibérica y que, por tanto, los efectos del calentamiento global deben ser tenidos en cuenta. Futuros trabajos deberían profundizar en una mejora de la exactitud y precisión de los métodos y datos empleados, ya sea incorporando un rango dinámico del factor $L$ de los índices de vegetación ajustados al suelo con apoyo de trabajo de campo, trabajando con sensores de mayor resolución espacial y relacionando explícitamente a través de series espacio-temporales las variables independientes y las variables respuestas.

\section{Agradecimientos}

Esta investigación forma parte del proyecto PALEOPINSAPO (Ref. CSO2017-83576-P).

\section{Referencias}

Alcaraz-Segura, D., Cabello, J. \& Paruelo, J., 2009. Baseline characterization of major Iberian vegetation types based on the NDVI dynamics. Plant Ecology, 202: 13-29. https://doi. org/10.1007/s11258-008-9555-2

Alcaraz-Segura, D., 2006. Caracterización del funcionamiento de los ecosistemas ibéricos mediante teledetección. Ecosistemas, 15: 113-117.

Badeck, F.W., Bondeau, A., Böttcher, K., Doktor, D., Lucht, W., Schaber, J. \& Sitch, S., 2004. Responses of spring phenology to climate change. New Phytologist, 162(2): 295-309. https://doi.org/10.1111/j.1469-8137.2004.01059.x

Baret, F., Jacquemoud, S. \& Hanocq, J. F. 1993. About the soil line concept in remote sensing. Advances in Space Research, 13(5): 281-284. https://doi.org/10.1016/0273-1177(93)90560-X

Bergmeier, E., 1998. Phenological Patterns of Oro-Mediterranean Vegetation in the White Mountains (Western Crete). En: Progress in Botanical Research (pp. 29-32). Dordrecht: Springer Netherlands. https://doi.org/10.1007/978-94-011-5274-7_5

Busetto, L. \& Ranghetti, L., 2016. MODIStsp: An R package for automatic preprocessing of MODIS Land Products time series. Computers \& Geosciences, 97: 40-48. https://doi. org/10.1016/j.cageo.2016.08.020

Cabello, J. \& Castro, A.J., 2012. Estado y tendencia de los servicios de los ecosistemas de Alta Montaña de Andalucía. Consejería de Medio Ambiente. Junta de Andalucía, Sevilla.

Catalan, J., Ninot, J.M. \& Aniz, M.M., 2017. The High Mountain Conservation in a Changing World. En: High mountain conservation in a changing world (pp. 3-36). Springer. https://doi.org/10.1007/978-3-319-55982-7

Chuvieco, E., 2008. Teledetección ambiental. Ariel, Barcelona.

Didan, K., 2015. MOD13Q1 MODIS/Terra Vegetation Indices 16-Day L3 Global 250m SIN Grid V006 [Data set]. NASA EOSDIS Land Processes DAAC. https://doi.org/10.5067/ MODIS/MOD13Q1.006 
Dullinger, S., Gattringer, A., Thuiller, W., Moser, D., Zimmermann, N. E., Guisan, A. \& Hülber, K., 2012. Extinction debt of high-mountain plants under twenty-first-century climate change. Nature Climate Change, 2(8): 619-622. https://doi. org/10.1038/nclimate1514

Eastman, J.R., Sangermano, F., Ghimire, B., Zhu, H., Chen, H., Neeti, N. \& Crema, S.C., 2009. Seasonal trend analysis of image time series. International Journal of Remote Sensing, 30(10): 2721-2726. https://doi.org/10.1080/01431160902755338

Eastman, J.R., Sangermano, F., Machado, E.A., Rogan, J. \& Anyamba, A., 2013. Global trends in seasonality of Normalized Difference Vegetation Index (NDVI), 1982-2011. Remote Sensing, 5(10): 4799-4818. https://doi.org/10.3390/rs5104799

Eastman, J.R., 2015. TerrSet: Geospatial Monitoring and Modeling Software. Worcester MA (USA): Clark University.

Engler, R., Randin, C.F., Thuiller, W., Dullinger, S., Zimmermann, N.E., Araújo, M.B. \& Guisan, A., 2011. 21 st century climate change threatens mountain flora unequally across Europe. Global Change Biology, 17(7): 2330-2341. https:// doi.org/10.1111/j.1365-2486.2010.02393.x

Ferreras, C. \& Fidalgo, C., 1991. Biogeografia y edafogeografia. Síntesis, Madrid.

Fort, M., 2015. Impact of climate change on mountain environment dynamics. Revue de Géographie Alpine, (103-2): 0-7. https://doi.org/10.4000/rga.2877

Gil, A., \& Olcina, J., 2017. Tratado de Climatología. Servicio de Publicaciones de la Universidad de Alicante, Alicante.

Gilabert, M.A., González-Piqueras, J., García-Haro, F.J. \& Meliá, J., 2002. A generalized soil-adjusted vegetation index. Remote Sensing of Environment, 82(2-3): 303-310. https:// doi.org/10.1016/S0034-4257(02)00048-2

Gómez-Zotano, J., Alcántara-Manzanares, J., Olmedo-Cobo, J.A. \& Martínez-Ibarra, E., 2015. La sistematización del clima mediterráneo: identificación, clasificación y caracterización climática de Andalucía (España). Revista de Geografía Norte Grande, 61: 161-180. https://doi.org/10.4067/ S0718-34022015000200009

Helman, D., 2018. Land surface phenology: What do we really 'see' from space? Science of the Total Environment, 618: 665-673. https://doi.org/10.1016/j.scitotenv.2017.07.237

Huber, U.M., Bugmann, H.K.M. \& Reasoner, M.A., 2005. Global Change and Mountain Regions. Springer Netherlands, Dordrecht. https://doi.org/10.1007/1-4020-3508-X

Huete, A., 1988. A soil-adjusted vegetation index (SAVI). Remote Sensing of Environment, 25(3): 295-309. https://doi. org/10.1016/0034-4257(88)90106-X

Huete, A.R., Hua, G., Qi, J., Chehbouni, A. \& van Leeuwen, W.J.D., 1992. Normalization of multidirectional red and NIR reflectances with the SAVI. Remote Sensing of Environment, 41(2-3): 143-154. https://doi.org/10.1016/0034-4257(92)90074-T

Huete, A., Justice, C. \& Liu, H., 1994. Development of vegetation and soil indices for MODIS-EOS. Remote Sensing of Environment, 49(3): 224-234. https://doi. org/10.1016/0034-4257(94)90018-3

Ibarra, P., 2003. Grandes dominios biogeográficos andaluces. En: Geografía de Andalucía (pp. 228-253). Ariel, Barcelona.

Jones, H.G. \& Vaughan, R.A., 2010. Remote Sensing of Vegetation: Principles, Techniques, and Applications. OUP Oxford, New York.

Lacoste, A. \& Salonon, R., 1973. Biogeografía. Oikos-Tau, Barcelona.

Laiolo, P. \& Obeso, J., 2017. Life-History Responses to the Altitudinal Gradient. En: High Mountain Conservation in a Changing World (pp. 253-287). https://doi. org/10.1007/978-3-319-55982-7 11

López, A., 2003. Unidad y variedad de Andalucía. En: Geografía de Andalucía (pp. 813-845). Ariel, Barcelona.
Ma, S., Pitman, A.J., Lorenz, R., Kala, J. \& Srbinovsky, J., 2016. Earlier green-up amplifies spring warming over Europe. Geophysical Research Letters, 46(3): 582-589. https:// doi.org/10.1002/2016GL068062

Mas, J., coord, 2011. Aplicaciones del sensor MODIS para el monitoreo del territorio. Universidad Nacional Autónoma de México, México DF.

Mesa Garrido, M.Á., 2016. Pinos autóctonos y conservación de bosques en Andalucía. Investigaciones Geográficas, 65: $97-$ 115. https://doi.org/10.14198/INGEO2016.65.06

Mesa, M.A., 2020. La Política Forestal en Andalucía: del Plan General de Repoblación Nacional al Plan Forestal Andaluz Granada (1938-2018). Cuadernos Geográficos, 59(1): 7898. https://doi.org/10.30827/cuadgeo.v59i1.8116

Myneni, R.B., Hall, F.G., Sellers, P.J. \& Marshak, A.L., 1995. The interpretation of spectral vegetation indexes. IEEE Transactions on Geoscience and Remote Sensing, 33(2): 481-486. https://doi.org/10.1109/TGRS.1995.8746029

Olmedo, J., 2012. Bosques relictos de Pinus sylvestris L. en la Sierra de Baza (Provincia de Granada, España): Análisis y cartografía del estado actual de la vegetación. Cuadernos Geograficos, 50(51): 37-61.

Pèlachs, A., Pérez-Obiol, R., Soriano, J.M., Cunill, R., Bal, M.-C. \& García-Codron, J.C., 2017. The Role of Environmental Geohistory in High-Mountain Landscape Conservation. En: High Mountain Conservation in a Changing World (Vol. 62, pp. 107-129). https://doi.org/10.1007/978-3-319-55982-7 5

Pérez-Luque, A.J., Sánchez-Rojas, C.P., Zamora, R., Pérez-Pérez, R. \& Bonet, F.J., 2015. Dataset of phenology of mediterranean high-mountain meadows flora (Sierra Nevada, Spain). PhytoKeys, 46(1): 89-107. https://doi.org/10.3897/ phytokeys.46.9116

Pita, M., 2003. El clima de Andalucía. En: Geografia de Andalucía (pp. 137-173). Ariel, Barcelona.

Qi, J., Chehbouni, A., Huete, A.R., Kerr, Y.H. \& Sorooshian, S., 1994. A modified soil adjusted vegetation index. Remote Sensing of Environment, 48(2): 119-126. https://doi. org/10.1016/0034-4257(94)90134-1

R Core Team, 2019. R: A language and environment for statistical computing. R Foundation for Statistical Computing, Vienna.

Rahbek, C., Borregaard, M.K., Colwell, R.K., Dalsgaard, B., Holt, B.G., Morueta-Holme, N. \& Fjeldså, J., 2019. Humboldt's enigma: What causes global patterns of mountain biodiversity? Science, 365: 1108-1113. https://doi. org/10.1126/science.aax0149

Reed, B.C., Schwartz, M.D., \& Xiao, X., 2009. Remote sensing phenology. En: Phenology of Ecosystem Processes, 231246. https://doi.org/10.1007/978-1-4419-0026-5 10

Richardson, A.D., Keenan, T.F., Migliavacca, M., Ryu, Y., Sonnentag, O. \& Toomey, M., 2013. Climate change, phenology, and phenological control of vegetation feedbacks to the climate system. Agricultural and Forest Meteorology, 169: 156-173. https://doi.org/10.1016/j.agrformet.2012.09.012

Rivas-Martinez, S., 1987. Memoria del mapa de series de vegetación de España. Ministerio de Agricultura, Pesca y Alimentación, Madrid.

Rixen, C. \& Wipf, S., 2017. Non-equilibrium in Alpine Plant Assemblages: Shifts in Europe's Summit Floras. En: High Mountain Conservation in a Changing World (Vol. 62, pp. 285-303). https://doi.org/10.1007/978-3-319-55982-7 12

Rodríguez, F., 2001. Las montañas: poblamiento y sistemas agrarios. En: Geografía de España (pp. 371-389). Ariel, Barcelona.

Roerink, G.J., Menenti, M. \& Verhoef, W., 2000. Reconstructing cloudfree NDVI composites using Fourier analysis of time series. International Journal of Remote Sensing, 21(9): 1911-1917. https://doi.org/10.1080/014311600209814 
Rouse, J., Haas, R. \& Schell, J., 1974. Monitoring the vernal advancement and retrogradation (greenwave effect) of natural vegetation. Texas A \& M University, 1-8.

Rubio, J.M., 1989. Biogeografia. Paisajes vegetales y vida animal. Síntesis, Madrid.

Rubio, J.M., 1992. Biogeografía. En: Geografía Física (pp. 425-578). Cátedra, Madrid.

Shafer, S.L., Bartlein, P.J. \& Whitlock, C. (2005). Understanding the Spatial Heterogeneity of Global Environmental Change in Mountain Regions. En Global Change and Mountain Regions (pp. 21-30). https://doi.org/10.1007/1-4020-3508-X_3

Schwartz, M.D., 2013. Phenology: An Integrative Environmental Science. Springer Netherlands, Dordrecht. https://doi. org/10.1007/978-94-007-6925-0

Valle, F. \& Navarro, F.B., 2004. Bioclimatología y biogeografía de Andalucía. En: Naturaleza. Botanica V. Proyecto Andalucía (pp. 18-42). Publicaciones Comunitarias, Sevilla.

Vandvik, V., Halbritter, A.H. \& Telford, R.J., 2018. Greening up the mountain. Proceedings of the National Academy of Sciences of the United States of America, 115(5): 833-835. https://doi.org/10.1073/pnas.1721285115

Wang, X., Piao, S., Xu, X., Ciais, P., Macbean, N., Myneni, R.B. \& Li, L., 2015. Has the advancing onset of spring vegetation green-up slowed down or changed abruptly over the last three decades? Global Ecology and
Biogeography, 24(6): 621-631. https://doi.org/10.1111/ geb.12289

Webster, G.L., 1961. The Altitudinal Limits of Vascular Plants. Ecology, 42(3): 587-590. https://doi.org/10.2307/1932249

White, M.A., de Beurs, K.M., Didan, K., Inouye, D.W., Richardson, A.D., Jensen, O.P. \& Lauenroth, W.K., 2009. Intercomparison, interpretation, and assessment of spring phenology in North America estimated from remote sensing for 1982-2006. Global Change Biology, 15(10): 2335-2359. https://doi.org/10.1111/j.1365-2486.2009.01910.x

Zhang, K., Kimball, J.S., Mu, Q., Jones, L.A., Goetz, S.J. \& Running, S.W., 2009. Satellite based analysis of northern ET trends and associated changes in the regional water balance from 1983 to 2005. Journal of Hydrology, 379(1-2): 92-110. https://doi.org/10.1016/j.jhydrol.2009.09.047

Zhang, X., 2012. Phenology and Climate Change. InTech. https://doi.org/10.5772/2146

Zhang, X., Friedl, M.A., Schaaf, C.B., Strahler, A.H., Hodges, J.C. F., Gao, F. \& Huete, A., 2003. Monitoring vegetation phenology using MODIS. Remote Sensing of Environment, 84(3): 471475. https://doi.org/10.1016/S0034-4257(02)00135-9

Zhang, X., Friedl, M., Tan, B., Goldberg, M. \& Yu, Y., 2012. Long-Term Detection of Global Vegetation Phenology from Satellite Instruments. En: Phenology and Climate Change (pp. 297-320). https://doi.org/10.5772/39197 